2. Dilution with distilled water is attended by less loss than is the case in dilution with very weak acids, and is reproducible for practical purposes if the dilution is not too great.

3. When an invertase solution is added to a solution containing sucrose, losses in activity due to dilution are less than when the invertase is diluted by a similar solution not containing sucrose.

4. The velocity of hydrolysis of sucrose by invertase is greater when a citrate buffer or acetate buffer is used as the source of hydrogen ions than when citric or acetic acid respectively is so used.

5. With different invertase preparations the magnitude of the losses in activity on dilution varies. Invertase solutions may be designated as more or less stable according to the magnitude of such losses in activity.

6. Both dilute and stock solutions of invertase lose strength on standing, the former faster than the latter. Stock solutions of the more stable invertase preparations lose activity more slowly than stock solutions of the less stable invertase preparations.

NEW YORK, N. Y.

[CONTRIBUTION FROM THE CHEMICAL LABORATORY OF HARVARd UNIVERSITY.]

\title{
ADDITION REACTIONS OF THE CARBONYL GROUP INVOLVING THE INCREASE IN VALENCE OF A SINGLE ATOM.
}

\author{
By James B. Conant.
}

Received April 29, 1921.

The addition reactions of the phosphorus halides, which have been described in previous papers, ${ }^{1}$ are of a different type from the usual addition reactions of simple or conjugated carbonyl compounds. They involve the formation of a cyclic compound by reason of the increase in valence of a single atom, while in most addition reactions of carbonyl compounds the reagent seems to have dissociated into two parts which subsequently become attached to the carbon and oxygen atoms. The Grignard reagent, hydrocyanic acid, and sodium bisulfite may be mentioned as common examples of this usual type of reaction.

$$
\mathrm{R}_{2} \mathrm{C}=\mathrm{O}+\mathrm{AB} \rightarrow \mathrm{R}_{2} \mathrm{C}-\mathrm{OB}
$$

These common reagents react with $\alpha, \beta$ unsaturated ketones and aldehydes in much the same manner. When they combine with the conjugated system, the two parts of the addendum attach themselves in the 1,4 position to the oxygen and carbon atoms. The molecule of the reagent apparently undergoes cleavage into the same fragments in the case of both 1,2 and 1,4 additions.

1 This Journal, 39, 2679 (1917); 42, 830, 2337 (1920); 43, 1665,1667 (1921). 
The 1,4 addition of phosphenyl chloride and the 1,2 and 1,4 additions of phosphorus trichloride do not fit into this classification; they seem to be examples of another sort of addition reaction. In these cases the addendum unites with the carbonyl group or conjugated system without cleavage and the phosphorus a.tom increases its apparent valence by two. The reaction is reversible.

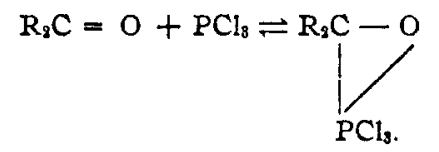

Such reactions involving the increase in valence of a single atom have been found to take place with a variety of simple carbonyl compounds, and also with unsaturated ketones. The phosphorus halide, also, may be varied. As shown in the experimental portion of this article, phosphorus tribromide readily adds in the 1,4 position to benzal-acetophenone. The equilibrium is more in favor of the addition product than with the trichloride. Several instances of the 1,4 addition of phosphenyl chloride have already been investigated and unpublished results obtained in this laboratory have shown that diphenoxy-chlorophosphine, monophenoxydichlorophosphine and diethoxy-dichlorophosphine behave in a similar manner. The reaction is thus general enough to warrant consideration from a theoretical standpoint.

Reactions of this special type are common in inorganic chemistry; for example, the reaction between ammonia and the halogen acids or between the trivalent phosphorus halides and chlorine. These reactions take place with the increase of valence of a single atom, and are for the most part reversible. The work of Werner and others provided a starting point for an interpretation of pentavalent nitrogen compounds, and the theory of the cubical atom as developed by G. N. Lewis ${ }^{2}$ and later by Langmuir ${ }^{3}$ has provided a satisfactory picture of the changes of valence of the elements in the fifth group of the periodic table. An application of this view has been helpful in the investigations just discussed and a further expansion of it leads to a general hypothesis in regard to most reactions of carbonyl compounds.

The Change from a Covalence of Three to a Covalence of Four.

According to Lewis' theory the three hydrogen atoms in ammonia are held to the nitrogen by the sharing of pairs of electrons of a group of eight. In ammonium chloride the four hydrogen atoms are likewise held by the sharing of electrons but the chlorine atom is not directly bound to the nitrogen; it is a negatively charged atom, and in solid ammonium chloride is held with the positively charged ammonium radical in a certain

${ }^{2}$ Lewis, This Journat, 38, 762 (1916).

${ }^{3}$ Langmuir, ibid., 41, 868 (1919). 
spatial arrangement by electrostatic forces. In solution both ions are free to move about. The linkage of hydrogen to nitrogen is a non-polar bond, (usually referred to as valence in organic chemistry); the chlorine atom is held by a polar bond (what is usually meant by valence in the chemistry of electrolytes). Similarly it seems probable that in phosphorus pentachloride, four chlorine atoms are held by non-polar bonds, and one by a polar bond. This is often represented thus: $\left[\left[\mathrm{PCl}_{4}\right]+\mathrm{Cl}^{-}\right]$.

The mechanism by which the non-polar valence (the covalence) of nitrogen and phosphorus in such compounds increases from three to four has been but little discussed. It involves the attachment of a positively charged atom or group by means of the two unshared electrons of the trivalent atom. In aqueous solution it is probably the hydrogen ion which is thus attracted. To explain the reaction in non-aqueous solutions or the addition of such non-electrolytes as methyl iodide to $\mathrm{RNH}_{2}$ or chlorine phosphorus trichloride, one may either posit the existence of ions, or conceive of molecules in which one pair of electrons is pulled toward one atom to such an extent that the positive nucleus of the other is exposed. Lewis ${ }^{2}$ has already pointed out these alternatives in the case of the halogens; there is some evidence which points to the existence of positive and negative halogen ions. Until more is known about this "ionization" of essentially non-polar substances no clear line can be drawn between the two cases. The addition reactions of inorganic chemistry, of the type under consideration, seem to depend on the attraction of the positive nucleus of some ion or "partially ionized" molecule, and the resulting formation of a polar compound;

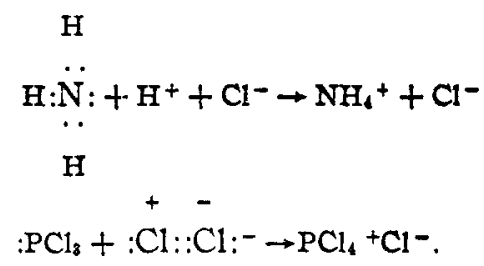

From the same point of view the carbon atom of the carbonyl group may act as if it were a positively charged ion. The positive nucleus of the carbon atom is more exposed in such compounds than in saturated compounds because of the drawing away of the two electrons which are shared with the oxygen octet. The positively charged nucleus will attract the unshared electrons of the central atom of such compounds as ammonia or phosphorus trichloride. When the molecules are drawn sufficiently close together an addition compound will be formed. The oxygen atom of this compound will be negatively charged, the nitrogen or phosphorus atom positively charged. The polar bond corresponding to the chloride ion of ammonium chloride will then be an "inner polar bond" stuch as exists in the amino acids; 


$$
\begin{aligned}
& \mathrm{R}_{2} \mathrm{C}: \ddot{\mathrm{O}}+\mathrm{PCl}_{3} \Longrightarrow \mathrm{R}_{2} \mathrm{C}: \ddot{\mathrm{O}} \\
& \begin{array}{l}
\because \ddot{\mathrm{Cl}}: \ddot{\mathrm{p}}: \ddot{\mathrm{Cl}}: \\
: \therefore \mathrm{Cl}:
\end{array}
\end{aligned}
$$

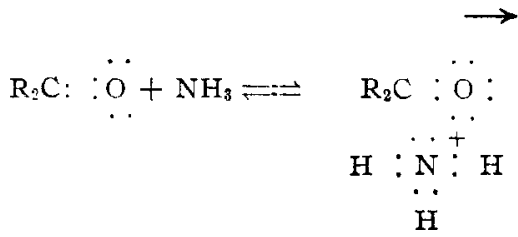

These reactions are obviously completely parallel to the addition of chlorine to phosphorus trichloride and of hydrogen chloride to ammonia.

In the case of phosphorus trichloride, the compound can easily shift its electrons so that the "inner polar bond" disappears and one chlorine atom is ionized. (Non-polar bonds are represented in the following by solid lines, polar bonds by dotted lines, or by a bracket.)
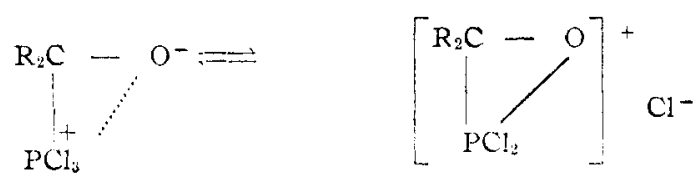

This new compound is equivalent to the pentavalent phosphorus halides such as $\mathrm{PCl}_{4}+\mathrm{Cl}^{-}$. The great similarity between the reactions of the addition compound of an aldehyde and phosphorus trichloride and of phosphorus pentachloride has already been emphasized; the greater reactivity of the pentavalent phosphorus halides is probably due to the fact that one of the chlorine atoms is held by a polar bond. The decomposition of the addition compound with water or acetic acid may be formulated as follows.

$$
\left.[]_{\mathrm{PCl}_{2}}^{\mathrm{R} \mathrm{C}^{2}}\right]_{\mathrm{O}}^{\mathrm{Cl}^{-}+\mathrm{HOH}} \rightarrow\left[\mathrm{OH}^{-}+\mathrm{HCl}\right.
$$

The acid chloride of the phosphonic acid can then further react and form the phosphonic acid $\mathrm{R}_{2} \mathrm{C}-\mathrm{POOH}$ which under suitable conditions is 
transformed ${ }^{4}$ to $\mathrm{R}_{2} \mathrm{CHOHPO}(\mathrm{OH})_{2}$. Similarly, as described in earlier papers, the 1,4 addition compound reacts with acetic anhydride producing a cyclic anhydride; with acetic acid an open chain phosphonic acid is formed.

This great reactivity of the chlorine atoms attached to the pentavalent phosphorus atom of the addition compound, is of great practical importance in carrying out all these addition reactions. It is possible to cause the reversible addition reactions to go to completion if cold acetic acid or acetic anhydride is used as a medium; if the proper temperature is maintained only the addition product will act with the acetic acid or anhydride. Very good yields of phosphonic acids (or their cyclic anhydrides) can thus be obtained from equimolecular amounts of phosphorus halide and unsaturated compound. However, in the initial addition reaction only about $30 \%$ of the two substances have combined as can be shown by rapidly decomposing the mixture with water, (see experimental part).

\section{Reactions of Nitrogen Compounds.}

In the case of the addition compounds of aldehydes with ammonia and substituted ammonias, such as hydroxylamine and phenylhydrazine, the initial product cannot rearrange in the same manner as the phosphorus trichloride addition product. Such a rearrangement would involve the formation of a negative hydrogen ion, which is obviously impossible. Rearrangement may take place, however, so that a hydrogen atom shifts to the oxygen atom, or water may be eliminated from the molecule forming an unsaturated compound (such as an oxime or phenyl hydrazone);
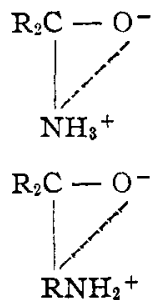

$\mathrm{R}_{2} \mathrm{C}-\mathrm{OH}$

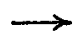

$\mathrm{NH}_{2}$

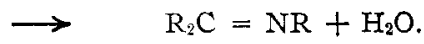

If neither of these possibilities can occur no stable addition product can be isolated as the initial reaction is easily reversible, and stops at some equilibrium point. However, in certain cases it may be that a compound of the inner salt type can be stable without rearrangement. E. A. Werner, ${ }^{5}$ or example, believes the formula for urea to be $\mathrm{NH}=\mathrm{C}_{3}$ and

4 This Journat, 42, 2337 (1920).

Werner, J. Chem. Soc., 113, 84 (1918). 
writes the reaction for its formation from cyanic acid and ammonia as

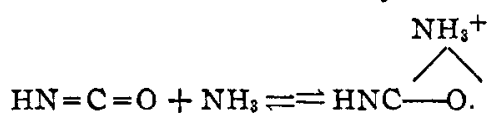

This is, of course, exactly the type of reaction we are here considering, and if Werner is correct in the interpretation of his experiments, the addition of ammonia to cyarlic acid is another case of an addition reaction of a carbonyl group which involves the increase in covalence of the addendum.

From this point of view: the great reactivity of trivalent nitrogen compounds towards aldehydes and ketones is due to the attraction of the unshared pair of electrons ox the nitrogen atom by the "exposed" nucleus of the carbon atom of the carbonyl group. It is exactly this attraction of the unshared electrons by the positive nucleus of the hydrogen ion which makes these same compounds weak bases. The two phenomena are thus paralle1.

\section{The Hypothesis Applied to Other Reactions.}

Many reactions which do not seem to involve the increase in valence of a single atom may also be interpreted by this hypothesis. Recent investigations ${ }^{6}$ seem to indicate that the Grignard reagent acts as if it were at least partially ionized; for example, into $\mathrm{CH}_{3}-$ and $\mathrm{MgI}{ }^{+}$. Such a negative ion would be attracted by the positive carbon atom of the carbonyl group; the product would be the salt of a very weak acid (the carbinol) which on treatment with water or acid would yield the hydrogen compound;

$$
\begin{gathered}
\mathrm{R}_{2} \mathrm{C}=\mathrm{O}+\mathrm{CH}_{3}-\mathrm{MgX}+\longrightarrow\left[\begin{array}{c}
\mathrm{R}_{2} \mathrm{C}-\mathrm{O} \\
1 \\
\mathrm{CH}_{3}
\end{array}\right]^{-} \mathrm{MgX}^{+} \\
{\left[\begin{array}{c}
\mathrm{R}_{2} \mathrm{C}-\mathrm{O} \\
\vdots \\
\mathrm{CH}_{3}
\end{array}\right]^{-} \mathrm{MgX}^{+}+\mathrm{HX} \rightarrow \underset{\mathrm{R}_{2} \mathrm{C}-\mathrm{OH}+\mathrm{MgX}_{2} .}{\left[\mathrm{CH}_{3}\right.}}
\end{gathered}
$$

Phosphorus pentachloride is another somewhat different reagent. Here the negative chlorine is attracted and the positive $\mathrm{PCl}_{4}$ group is held to the resulting complex by a polar bond. Such a compound might undergo an irreversible intramolecular change with the formation of $\mathrm{POCl}_{3}$ and a dichloride;

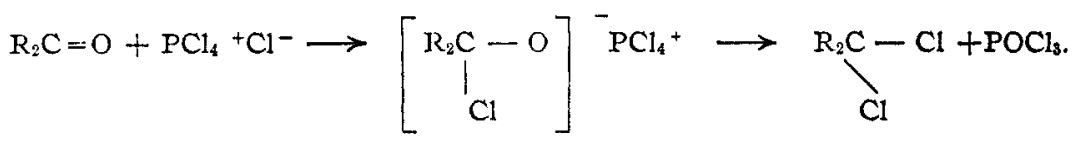

ti. This Jovrat, 39, 82 (1917). 
A similar mechanism has often been suggested for this reaction and agrees with the results obtained by the action of this reagent on conjugated systems. It is in some ways the exact opposite of the action of the phosphorus trichloride which has been discussed above. The difference between these reagents illustrates the contrast between trivalent and pentavalent phosphorus compounds.

In conjugated systems there are three carbon atoms in which the positive nucleus is exposed by reason of the sharing of two electrons in an octet. The addendum with its negative electrons or negative charge may be attracted to any one of these three.

$$
\begin{aligned}
& \text { >C: : } \mathrm{C}^{1}: \mathrm{C}^{\prime}:: \ddot{\mathrm{O}} \\
& \begin{array}{llll}
1 & 2 & 3 & 4
\end{array}
\end{aligned}
$$

Only the first and third carbon atoms, however, have the possibility of attaching the addendum in such a way that the oxygen atom acquires a negative charge and becomes part of the "inner polar bond" by completing its octet. Thus, a reaction completely parallel to that with the simple carbonyl group can take place either by the addition on the third carbon atom (1,2 addition) or on the first carbon atom (1,4 addition). Which one of these reactions will predominate will depend on their relative speeds which in turn are probably influenced by the positive force fields of the nuclei of the two carbon atoms and steric hindrance. The case of 1,4 addition of phosphorus trichloride would be represented thus:

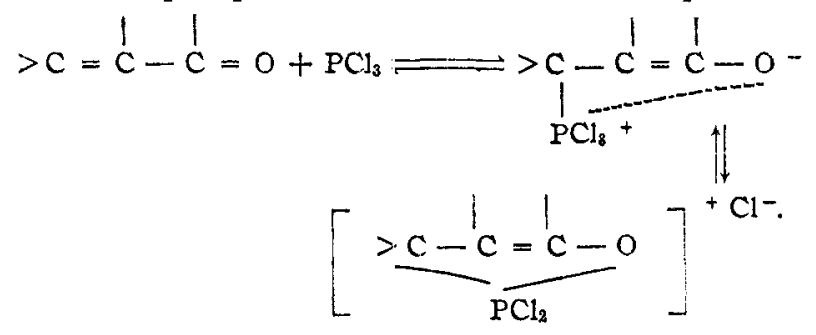

To sum up, the present hypothesis supposes that the reactivity of carbonyl compounds (both simple and conjugated) depends on the attraction of the positive force fields of the carbon atom for some atom having unshared electrons or some negative ion. The initial reaction in the case of trivalent nitrogen and phosphorus compounds leads to the formation of a compound with an "inner polar bond." The reaction is easily reversible. The position of the equilibrium and the speed of the reaction would seem to depend on the electrostatic forces between the atoms and on the ability of the molecules to come into close proximity (steric hindrance). The final stable product of these reactions is formed by a further irreversible rearrangement or reaction of the addition compound; the nature of such a product and its speed of formation will depend not only on the 
equilibrium of the initial reaction but on the speeds of the subsequent reactions. By analogy with certain inorganic substances and reactions it has been possible to formulate roughly some of these factors in the case of the action of the phosphorus halides. Until more quantitative evidence is at hand it will not be profitable to discuss the influence of various groups on the course of such reactions, although eventually this hypothesis should be useful in correlating many of the facts concerning the so-called "reactivity" of carbonyl compounds.

The reactions of ethylene oxides ${ }^{7}$ are readily formulated by means of this same hypothesis. The electrons of the carbon atoms are drawn away in the formation of a three-membered ring leaving the positive nucleus of the carbon atoms exposed. The force fields of these carbon nuclei would attract the same sort of molecules as in the case of carbonyl compounds. The resulting substances could undergo the same sort of rearrangement; for example with ammonia;

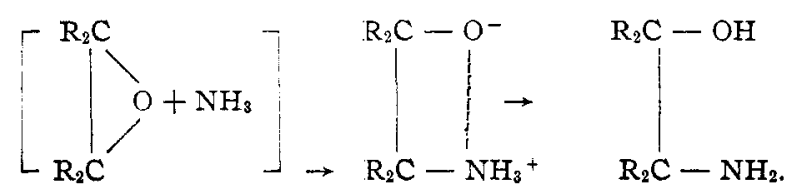

This electronic interpretation of the facts summarized by Baeyer's strain theory has already been pointed out by Langmuir. In larger rings the electrons are drawn away to a less extent and four-, five- and six-membered rings are therefore less reactive.

In conclusion the writer wishes to express his great indebtedness to Dr. E. Q. Adams of the Bureau of Chemistry for having pointed out to him in 1917 the possibility of applying Professor Lewis' ideas to the addition reactions of phosphorus trichloride and to similar problems in organic chemistry.

\section{Experimental.}

The Equilibrium between Phosphorus Trichloride and Benzal-acetophenone.-The addition reaction between benzaldehyde and phosphorus trichloride has already been shown ${ }^{8}$ to be reversible; a definite equilibrium point is reached and the determination of this equilibrium has shown that the reaction follows the usual law of mass action. The method used in that case was to decompose the reaction mixture with water and determine the amount of phosphorus acid formed by the hydrolysis of the uncombined trichloride. Both the addition product and the trichloride react so rapidly with water that little if any shifting of the equilibrium takes place during hydrolysis. The results obtained showed that when

7 The action of the phosphorus halides on such compounds is being investigated.

8 ThIS JOURNAI, 42, 2337 (1920). 
equimolecular amounts of benzaldehyde and phosphorus trichloride are brought together, equilibrium is obtained when $30 \%$ of the material has combined.

It was of interest to obtain some similar values for the 1,4 addition of phosphorus trichloride. This was done in a rough way by determining the amount of phosphonic acid formed (which is insoluble in water) and the amount of ketone left unchanged when the reaction mixture was decomposed with water. The procedure was as follows.

Five g. of benzal-acetophenone, $0.024 \mathrm{~g}$. mol. (carefully dried in a vacuum over phosphorus pentoxide), and $3.4 \mathrm{~g}$. of phosphorus trichloride $(0.025 \mathrm{~g} . \mathrm{mol}$.) were mixed together in a glass tube which was then sealed. The solid ketone soon dissolved almost completely in the trichloride. The mixture was allowed to stand for a definite time at room remperature $\left(25^{\circ}\right)$. The tube was then opened and the contents quickly stirred into $250 \mathrm{cc}$. of water. The water solution was carefully extracted with ether and the phosphonic acid then removed from the ethereal layer by extraction with sodium hydroxide. Evaporation of the ether yielded the unchanged ketone. The phosphonic acid was obtained by acidifying the sodium hydroxide solution and extracting with ether. The amount of recovered ketone and phosphonic acid in every case accounted for at least $95 \%$ of the original material. The following results were obtained.

$\begin{array}{cccc}\text { Hours. } & \text { Acid g. } & \text { Ketone equivalent. } & \text { Per cent. reacted. } \\ 1 & 1.5 & 1.1 & 22 \\ 2 & 1.6 & 1.2 & 24 \\ 3 & 1.5 & 1.1 & 22 \\ \left.1 \text { (4 mols } \mathrm{PCl}_{4}\right) & 3.2 & 2.3 & 46\end{array}$

These figures while only approximate show that the reaction comes to a definite equilibrium in a comparatively short time. When a large excess of one of the materials is employed (last experiment) more addition product is formed. The recovered ketone and phosphonic acid were both very pure as shown by their sharp melting points: this is evidence that no side reactions are appreciable in this length of time. When the components are left in contact for many days, however, other reactions take place; the acid material becomes impure and the non-acid material is no longer unchanged ketone but some oily substance.

The Addition of Phosphorus Tribromide to Benzal-acetophenone.Phosphorus tribromide combines with benzal-acetophenone even more readily than the trichloride. The reaction was investigated by bringing the materials together and after a given time decomposing with water as described in the experiment with the trichloride. In this case, however, $\beta$-bromobenzyl-acetophenone was obtained as the non-acid material instead of the unchanged ketone. This was probably due to the addition of hydrobromic acid to the unsaturated ketone during the decomposition of the mixture with water; an oily mass is at first formed which it takes some minutes to decompose completely by agitating with water. When the reaction mixture was poured into dil. sodium hydroxide solution instead of water, most of the non-acid material was unchanged ketone. 
The following results were obtained with equimolecular quantities.

$\begin{array}{cccc}\text { Hours. } & \text { Acid g. } & \text { Ketone equivalent. } & \text { Per cent. reacted. } \\ 1 & 3.7 & 2.6 & 52 \\ 1 & 3.2 & 2.2 & 44 \\ 2 & 3.6 & 2.5 & 50 \\ 4 & 3.6 & 2.5 & 50\end{array}$

It is evident that the equilibrium is much more in favor of the addition product with phosphorus tribromide than with phosphorus trichloride.

Reaction in Acetic Acid.--5 g. of benzal-acetophenone was mixed with $6.8 \mathrm{~g}$. of phosphorus tri-bromide (10\% excess) and $7 \mathrm{~g}$. of acetic acid. The ketone dissolved and the reaction mixture then became quite warm while fumes of acetyl bromide were apparent. After standing for 3 hours the mixture was poured into water and the materials were separated in the usual way. $6.2 \mathrm{~g}$. of pure phosphonic acid was obtained $(88 \%)$ and $0.5 \mathrm{~g}$. of unchanged ketone.

\section{Summary.}

1. The addition reactions of the phosphorus halides with carbonyl compounds are of a different type from the reactions of most reagents with these substances. They involve the formation of a cyclic compound with the increase in valence of a single atom while the more usual reactions take place with the cleavage of the reagent into two parts. The reaction is reversible.

2. This new type of reaction is analogous to addition reactions of inorganic chemistry such as the addition of the halogen acids to ammonia.

3. Lewis' theory of the cubical atom has been applied to this problem. The positive nucleus of the carbon atom of the carbonyl group is exposed by the drawing away of the electrons of the double bond. This exposed nucleus behaves in addition reactions like a positively charged atom and unites with atoms having unshared electrons (the phosphorus atom in the trichloride and the nitrogen atom in ammonia).

4. The resulting addition compounds may undergo various irreversible intramolecular changes or react irreversibly with other reagents. In such cases stable products will be formed.

5. This hypothesis is applied to the 1,2 and 1,4 addition of phosphorus halides, ammonia and its derivatives, the Grignard reagent and phosphorus pentachloride. The reactions of ethylene oxide are also considered.

6. Phosphorus tribromide combines with benzal-acetophenone in the 1,4-position, the reaction being parallel to that with the trichloride. The equilibrium has been measured for both substances; it is more in favor of the addition product in the case of the tribromide than with the trichloride. 